\title{
Recognition failure when recognition targets and recall cues are identical
}

\author{
GREGORY V. JONES \\ University of Warwick, Coventry, England \\ and \\ JOHN M. GARDINER \\ City University, London, England
}

\begin{abstract}
An experiment is reported in which the recognition and recall of target words were compared when recall was cued by other words that were either identical or not identical to the targets. Two types of nonidentical cue were used-semantic cues and associate cues (e.g., down and hope, respectively, for the target HIGH; in this case, the identical cue was, of course, high). The results are shown to have two principal implications. First, the results are consistent with the dual-route model of recall and, in particular, support earlier indications that direct and indirect routes to recall may be exclusive in operation. Second, they demonstrate that the empirical Tulving-Wiseman law that describes the relation between recognition and recall over a wide range of conditions does not hold for identical cues, thereby providing further confirmation of its established range of applicability.
\end{abstract}

Common experience indicates that, where memory is fallible, people are usually more likely to be able to recognize information when it is re-presented to them than they are likely to be able to recall the same information from mental storage. But the converse pattern of fallibility is also widespread. When a person has been presented with a number of different target items to remember, it is generally found that a certain proportion of the targets can be recalled but not recognized. The existence of such instances of "recognition failure" is enough to cast severe doubt on any theory that claims that all recall requires a component process of recognition. Recognition failure thus initially came to prominence as evidence against a strong version of the generation-recognition theory of recall. The latter theory would assert that all recall involves, first, mentally generating a number of possible candidates and, second, recognizing the candidate that corresponds to the correct target item. Although the existence of recognition failure appears to render this strong version of generation-recognition theory untenable, several weaker versions of the theory have also been proposed. Watkins and Gardiner (1979) have marshalled arguments against a number of these. However, one possibility that they did not exclude is that the recall of some items occurs via an indirect, generation-recognition route

The authors are grateful to Endel Tulving for helpful discussion. Address correspondence to either Gregory V. Jones, Department of Psychology, University of Warwick, Coventry CV4 7AL, England, or John M. Gardiner, Memory \& Cognition Research Group, City University, Northampton Square, London EC1V OHB, England. (in which recall is dependent upon recognition), while the recall of other items occurs by a second, direct route, in which recall is independent of recognition. The existence of recognition failure is clearly no problem for this dualroute model, since all such instances can be attributed to direct recall. However, its success on the simple issue described thus far is a hollow one, since it is bought merely at the price of a more complicated model. The value of the model is demonstrated only if it can be shown to account for more complex aspects of the relation between recognition and recall, which in principle afford opportunities for empirical disconfirmation. A relevant test case is provided by the data embraced by what in previous articles (e.g., Gardiner, 1988, 1989; Jones, 1980,1982 ) we have termed the Tulving-Wiseman law.

The Tulving-Wiseman law summarizes the results of a considerable number of studies in which the degree of association between recognition and recall has been quantitatively assessed. Tulving and Wiseman (1975) pointed out that observed estimates of the probability of recognition conditional upon recall are, in general, an approximate function of the overall probability of recognition. Specifically, the observed probability of recognition given recall, $P(\mathrm{Rn} \mid \mathrm{Rc})$, is approximated by the Tulving-Wiseman function, $P(\mathrm{Rn} \mid \mathrm{Rc})_{\mathrm{Tw}}$, which is obtained from the overall probability of recognition, $P(R n)$, as follows:

$$
P(\mathrm{Rn} \mid \mathrm{Rc})_{\mathrm{Tw}}=P(\mathrm{Rn})+1 / 2\left[P(\mathrm{Rn})-P(\mathrm{Rn})^{2}\right] .
$$

Now it would, of course, be surprising if for any individual set of data, there were perfect agreement between the observed estimate of $P(R n \mid R c)$ and the Tulving-Wise- 
man value $P(R n \mid R c)_{\text {Tw. Two different approaches may }}$ be adopted to explain the origin of observed discrepancies between the two measures.

The first approach posits that $P(\mathrm{Rn} \mid \mathrm{Rc})_{\text {Tw }}$ is always only a first approximation to $P(R n \mid R c)$, and that discrepancies between the two values-whether large or small-are themselves predictable on the basis of appropriate theory. Jones (1978) adopted this approach and showed how it is possible to predict the magnitude and direction of discrepancies between $P(\mathrm{Rn} \mid \mathrm{Rc})$ and $P(\mathrm{Rn} \mid \mathrm{Rc})_{\mathrm{Tw}}$ on the basis of the dual-mechanism model of recall described previously. Developing this model further, Jones (1987) showed that three different forms of the dual-route model may be distinguished, depending on the nature of the relation between the two routes. The probability of successfully generating the target item within the generation-recognition route may be estimated from the data in three different ways, depending on whether the relation between the generation process and the direct recall process is postulated to be one of mutual exclusivity, of independence, or of redundancy. The three generation probabilities given by Jones $(1987$, p. 232$)$ may be expressed in terms of $P(\mathrm{Rn}), P(\mathrm{Rn} \mid \mathrm{Rc})$, and $P(\mathrm{Rc})$ - the overall probability of recall-as

$$
\begin{aligned}
P(\mathrm{Rc})[P(\mathrm{Rn} \mid \mathrm{Rc})- & P(\mathrm{Rn})] /\{P(\mathrm{Rn})[1-P(\mathrm{Rn})]\}, \\
P(\mathrm{Rc})[P(\mathrm{Rn} \mid \mathrm{Rc})- & P(\mathrm{Rn})] /\{P(\mathrm{Rn})[1-P(\mathrm{Rn}) \\
& -P(\mathrm{Rc})+P(\mathrm{Rc}) P(\mathrm{Rn} \mid \mathrm{Rc})]\},
\end{aligned}
$$

and

$$
P(\mathrm{Rc}) P(\mathrm{Rn} \mid \mathrm{Rc}) / P(\mathrm{Rn}),
$$

for relations of mutual exclusivity, independence, and redundancy, respectively. Mutual exclusivity means that an item is recalled in practice by only one or the other of the two routes. Independence means that whether an item is recalled by one route does not affect whether or not it is also recalled by the other route. Redundancy means that whenever an item is directly recalled, it is also always generated. For the sake of completeness, it may be mentioned that the probability of direct recall can also be expressed in a similar manner as

$$
P(\mathrm{Rc})[1-P(\mathrm{Rn} \mid \mathrm{Rc})] /[1-P(\mathrm{Rn})] .
$$

However, the direct recall probability does not differ for the three types of theoretical relation, and was not studied in the experiment reported here.

The second approach posits, in contrast, that $P(\mathrm{Rn} \mid \mathrm{Rc})_{\mathrm{Tw}}$ is generally an accurate estimate of $P(\mathrm{Rn} \mid \mathrm{Rc})$, with small discrepancies deriving simply from random error of measurement. Only in the minority of cases for which there are large discrepancies do processes occur that are qualitatively different from those normally leading to Tulving-Wiseman values. Large discrepancies of this type have been observed when cues and targets are poorly integrated (e.g., Bartling \& Thompson, 1977; Begg, 1979; Gardiner \& Tulving, 1980), when cues and targets are category names and exemplars (Nilsson \&
Shaps, 1980), and when cues are descriptive phrases and targets are unique names of famous people (Muter, 1984). In all of these cases, values of $P(R n \mid R c)$ were considerably higher than those of $P(R n \mid R c)_{\text {Tw. It appears that }}$ these discrepancies arise when the recall cue does not provide information about the occurrence of the target in the study list beyond that provided by the target itself in recognition (see Flexser \& Tulving, 1978; Gardiner, 1988; Nilsson, Dinniwell, \& Tulving, 1987; Nilsson, Law, \& Tulving, 1988; Tulving, 1983). If this is so, one further case in which discrepancies between $P(R n \mid R c)$ and $P(\mathrm{Rn} \mid \mathrm{Rc})_{\text {Tw }}$ would be expected to arise is that in which the recall cue is an identical copy of its target. However, in an earlier study by Tulving (1974), which included such a condition, no such discrepancy was observed. The reason for what appears retrospectively to be a surprising result may be that since the subjects knew that some recall cues were identical to targets, they simply responded with some of these words as guesses. For such words, recognition and recall would be independent, potentially driving down overall values of $P(\mathrm{Rn} \mid \mathrm{Rc})$ that might otherwise have been

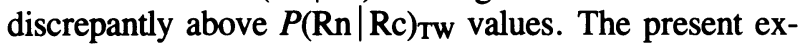
periment was designed to test this hypothesis by comparing conditions in which instructions either encouraged or discouraged such guessing. It was expected that substantial discrepancies between $P(R n \mid R c)$ and $P(R n \mid R c)_{\text {Tw }}$ values would be observed only in the latter case.

The present experiment also allowed the first approach's dual-route model of recall to be tested. Recall cues identical to targets should give rise to probabilities of generating the targets that are high relative to those for other types of cue. It was shown by Jones (1987) that this was indeed the case when the mutual exclusivity form of the dual-route model was applied to the Tulving (1974) data, and a similar pattern of results was expected in the present experiment. It may be noted that the tests of the two different approaches to recognition failure are essentially independent of each other. That is to say, it is in principle possible that the data to be obtained will prove to be compatible with both approaches, with one or the other of the approaches only, or with neither of the approaches.

\section{METHOD}

\section{Subjects}

The subjects were 60 undergraduates at the University of Toronto, divided randomly into four groups, tested up to 3 at a time.

\section{Materials}

All subjects studied a critical list of 24 A-B word pairs, and were tested first for recognition of the B items and second for recall of the $B$ items when the A items were presented as cues. Stimulus materials were taken from those used previously by Tulving (1974). For generality, two of the subject groups received a list containing a mixture of three different types of word pairs, whereas the other two groups received unmixed lists. Similarly, one of the groups receiving a mixed list had lures added to the $\mathrm{A}$ items provided as recall cues, whereas the other groups were provided with unlured recall cues.

The first group-24 subjects-was assigned to a mixed, lured condition. Their experimental list was composed of equal numbers of each of three sets of A-B pairs. In the semantic set, the target was semanti- 
cally related to the cue. In the associate set, the target was a free associate of the cue with a mean normative strength of approximately $1 \%$. In the identical set, the target was identical to the cue. In the recall test, the subjects received all $24 \mathrm{~A}$ words as cues, together with eight lures. The latter were selected from among the distractors in the recognition test.

The second group- 12 subjects - was assigned to a mixed, unlured condition. It differed from the preceding condition only in that the lure cues were omitted from the recall test.

The two remaining groups -12 subjects each-were both assigned to the unmixed, unlured condition. For one group, all B words were associates of paired A words, whereas for the other group, all B words were identical to paired $\mathrm{A}$ words.

\section{Procedure}

All subjects received a practice list of 16 pairs followed directly by a recall test. In each case, the A-B pair relations were similar to those in the subsequent critical list. The A and B items were all in lower and upper case, respectively, and were presented at a 2 -sec rate. The subjects in the mixed, lured and mixed, unlured conditions were informed beforehand that some A and B words would be identical; those in the identical-cue group of the unmixed, unlured condition were informed that all $\mathrm{A}$ and $\mathrm{B}$ words would be identical.

Following a 15-min filled interval, the subjects received two successive recognition tests. In a guessing discouraged test, they were asked to go through three columns of words, circling only those they were certain they had been presented with. Then, in a guessing encouraged test, they went through them again with a different-color pen, making a forced response on each row that did not have a previously circled word.

Finally, the subjects received two successive recall tests. In a guessing discouraged test, they were asked to work down the list of A words, writing down only B words that they were certain they had been presented with. Then, in a guessing encouraged test, they worked down the list again with a different-color pen. This time, they were asked to write a response for each $\mathrm{A}$ word that did not already have one, even if it had to be a guess. The subjects in the fourth group (i.e., those with identical cues in the unmixed, unlured condition) differed from the other three groups in that they were not presented with a list of A words.

\section{RESULTS}

Table 1 summarizes the data obtained in this experiment. It shows the observed overall probabilities of recognition and recall, together with the observed and TulvingWiseman values for the probability of recognition conditional upon recall. The level of false positives was no more than $3 \%$ in any condition of the guessing discouraged recognition test.
Table 1 also shows the difference between estimated values of $P(R n \mid R c)$ and of $P(R n \mid R c)_{\text {Tw. It can be seen }}$ that the only substantial discrepancies between the two sets of values occurred for identical cues in each of the guessing discouraged conditions, irrespective of whether the word lists were mixed or unmixed, and of whether the cues were lured or unlured. For these identical cues, observed values of $P(\mathrm{Rn} \mid \mathrm{Rc})$ were considerably greater than corresponding $P(R n \mid R c)_{\text {Tw }}$ values. As expected, however, similar unusually high levels of dependency between recall and recognition were not observed when guessing was encouraged.

In applying the dual-route model to these results, it is appropriate to consider only the guessing discouraged data, since the effects of guessing are not included in the model as formulated (although it could be extended to include guessing). Table 2 shows the estimated probability of generating a target given its cue, according to the three different assumptions of exclusivity, independence, and redundancy. It can be seen that, for each of the experimental conditions (mixed, lured; mixed, unlured; and unmixed, unlured), the generation probability was consistently greater for identical cues than for semantic and associate cues when exclusivity was assumed, although as expected, this was not so when independence or redundancy was assumed. It is noteworthy that even though the generation probability was highest for identical cues, Table 1 shows that the overall recall probability (to which it contributes) was lowest for identical cues in each of the three conditions.

\section{DISCUSSION}

The results of the present experiment provide support for specific theoretical formulations within two different approaches to understanding recognition failure.

One approach asserts at the most general level that any empirical estimate of the probability of recognition conditional upon recall will deviate systematically to a greater or lesser extent from the first-approximation value provided (as a function of overall recognition probability) by the Tulving-Wiseman law. Within this general approach, the present results were shown to agree with those analyzed by Jones (1987) in conform-

Table 1

Probabilities of Recognition, Recall, and Recognition Given Recall

\begin{tabular}{|c|c|c|c|c|c|c|c|c|}
\hline \multirow[b]{2}{*}{ Measure } & \multicolumn{3}{|c|}{ Mixed, Lured } & \multicolumn{3}{|c|}{ Mixed, Unlured } & \multicolumn{2}{|c|}{$\begin{array}{c}\text { Unmixed, } \\
\text { Unlured }\end{array}$} \\
\hline & $\mathbf{S}$ & A & I & $S$ & $\mathbf{A}$ & I & $\mathbf{A}$ & I \\
\hline \multicolumn{9}{|c|}{ Guessing Discouraged } \\
\hline Recognition (Rn) & .542 & .500 & .385 & .500 & .375 & .208 & .385 & .573 \\
\hline Recall (Rc) & .630 & .594 & .391 & .552 & .604 & .219 & .517 & .413 \\
\hline $\mathbf{R n} \mid \mathbf{R c}$ & .603 & .588 & .772 & .623 & .518 & .667 & .524 & .874 \\
\hline $\mathrm{Rn} \mid \mathrm{Rc}_{\mathrm{TW}}$ & .666 & .625 & .503 & .625 & .492 & .290 & .503 & .695 \\
\hline$\left(\mathbf{R n}|\mathbf{R c}-\mathbf{R n}| \mathbf{R c}_{\mathrm{TW}}\right)$ & -.063 & -.037 & .269 & -.002 & .026 & .377 & .021 & .179 \\
\hline \multicolumn{9}{|c|}{ Guessing Encouraged } \\
\hline Recognition (Rn) & .859 & .771 & .719 & .813 & .760 & .531 & .764 & .823 \\
\hline Recall (Rc) & .714 & .630 & .563 & .635 & .625 & .542 & .563 & .573 \\
\hline $\mathbf{R n} \mid \mathbf{R c}$ & .875 & .819 & .851 & .887 & .800 & .596 & .838 & .946 \\
\hline $\mathbf{R n} \mid \mathbf{R c}_{\mathbf{T w}}$ & .920 & .859 & .820 & .889 & .851 & .656 & .854 & .896 \\
\hline$\left(\mathbf{R n}|\mathbf{R c}-\mathbf{R n}| \mathbf{R c}_{\mathbf{T W}}\right)$ & -.045 & -.040 & .031 & -.002 & -.051 & -.060 & -.016 & .050 \\
\hline
\end{tabular}

Note-S, A, and I refer to semantic, associate, and identical cue types, respectively. 
Table 2

Probabilities of Generation for a Dual-Route Model with Three Types of Relation Between Generation and Direct Recall

\begin{tabular}{|c|c|c|c|c|c|c|c|c|}
\hline \multirow[b]{2}{*}{ Relation } & \multicolumn{3}{|c|}{ Mixed, Lured } & \multicolumn{3}{|c|}{ Mixed, Unlured } & \multicolumn{2}{|c|}{$\begin{array}{l}\text { Unmixed } \\
\text { Unlured }\end{array}$} \\
\hline & $S$ & A & I & $S$ & A & I & A & I \\
\hline Exclusivity & .156 & .208 & .640 & .272 & .369 & .610 & .303 & .508 \\
\hline Independence & .342 & .408 & .748 & .466 & .691 & .672 & .506 & .579 \\
\hline Redundancy & .701 & 698 & .784 & .688 & .835 & .702 & .704 & .630 \\
\hline
\end{tabular}

Note-S, A, and I refer to semantic, associate, and identical cue types, respectively.

ing to the dual-route model of recall. According to the version of the model supported by both studies, an individual target word may be recalled either via a direct route or via an indirect, generation-recognition route, but not via both routes. The direct and indirect routes were identified by Jones (1983) as utilizing two different types of graph structure within a semantic network-fragments and schemas, respectively. With the speeded presentation in these experiments, it is plausible that as soon as a subject has formed a satisfactory encoding in either fragment or schema format, he or she switches attention to another item, giving rise to the observed mutual exclusivity of the two retrieval routes at the level of the individual item.

The second approach asserts at the most general level that systematic deviations from the Tulving-Wiseman function occur only under specific experimental conditions. Again, the present results agree with previous findings showing that substantial deviations from the Tulving-Wiseman function occur when the cue does not provide useful information additional to that provided by the target itself. Here, a result of this kind was observed when cues were identical to targets although, as anticipated, it was found that this deviance was obscured when guessing was not discouraged.

Finally, it should perhaps be noted that the two approaches explored in this article-one concerned directly with the Tulving-Wiseman law and its exceptions, and the other viewing the same phenomena from the perspective of the dual-route theory of recall-may be complementary rather than conflicting in nature. Their relation appears to be analogous to that holding between, say, Boyle's law and the kinetic theory of gases. In each case, the theory (dual-route or kinetic) aims to provide a principled explanation of the empirical generalization expressed by the relevant law (Tulving-Wiseman or Boyle), and also of the particular circumstances that lead to the breakdown of that generalization. Depending on one's purposes, it is appropriate at any one time to conceptualize particular situations in terms of either the law or the theory or, as in the present case, in terms of both.

\section{REFERENCES}

Bartling, C. A., \& Thompson, C. P. (1977). Encoding specificity: Retrieval asymmetry in the recognition failure paradigm. Journal of Experimental Psychology: Human Learning \& Memory, 3, 690-700.

BEGG, I. (1979). Trace loss and the recognition failure of unrecalled words. Memory \& Cognition, 7, 113-123.

FleXser, A. J., \& TUlVING, E. (1978). Retrieval independence in recognition and recall. Psychological Review, 85, 153-171.

GARDINER, J. M. (1988). Recognition failures and free-recall failures:
Implications for the relation between recall and recognition. Memory \& Cognition, 16, 446-451.

Gardiner, J. M. (1989). The Tulving-Wiseman law and exceptions to it. In A. F. Bennett \& K. M. McConkey (Eds.), Cognition in individual and social contexts. Amsterdam: Elsevier.

GaRDINER, J. M., \& TulviNG, E. (1980). Exceptions to recognition failure of recallable words. Journal of Verbal Learning \& Verbal Behavior, 19, 194-209.

JONES, G. V. (1978). Recognition failure and dual mechanisms in recall. Psychological Review, 85, 464-469.

JONES, G. V. (1980). On the dual-mechanism theory of recognition failure: A reply to Begg. Memory \& Cognition, 8, 180-181.

JONES, G. V. (1982). Tests of the dual-mechanism theory of recall. Acta Psychologica, 50, 61-72.

JONES, G. V. (1983). Structure of the recall process. Philosophical Transactions of the Royal Society of London, B302, 373-385.

JONES, G. V. (1987). Independence and exclusivity among psychological processes: Implications for the structure of recall. Psychological Review, 94, 229-235.

MUTER, P. (1984). Recognition and recall of words with a single meaning. Journal of Experimental Psychology: Learning, Memory, \& Cognition, 10, 198-202.

Nilsson, L.-G., DinNiwell, M., \& TUlVING, E. (1987). Recognition failure of categorized words. Memory \& Cognition, 15, 389-396.

Nilsson, L.-G., LAw, J., \& Tulving, E. (1988). Recognition failure of recallable unique names. Journal of Experimental Psychology: Learning, Memory, \& Cognition, 14, 266-277.

Nilsson, L.-G., \& ShaPS, L. P. (1980). A functional view of memory. In F. Klix \& J. Hoffman (Eds.), Cognition and memory: Interdisciplinary research of human memory activities (pp. 40-46). Berlin: Deutscher Verlag der Wissenschaften.

TUlVING, E. (1974). Recall and recognition of semantically encoded words. Journal of Experimental Psychology, 102, 778-787.

Tulving, E. (1983). Elements of episodic memory. New York: Oxford University Press.

Tulving, E., \& WisEmaN, S. (1975). Relation between recognition and recognition failure of recallable words. Bulletin of the Psychonomic Society, 6, 79-82.

WATKINS, M. J., \& GARDINER, J. M. (1979). An appreciation of generate-recognize theory of recall. Journal of Verbal Learning \& Verbal Behavior, 18, 687-704.

(Manuscript received August 31, 1989.) 